\title{
Fishing for Food: Values and benefits associated with coastal infrastructure
}

Researchers: Cassandra Nieman, Alexie Rudman, Maggie Chory, Grant Murray, Luke Fairbanks, Lisa Campbell

The accompanying files in this archive provide data collected from semi-structured informal interviews conducted as part of the project "Fishing for Food: Values and benefits associated with coastal infrastructure" (visit the Duke University Master's Project, https://hdl.handle.net/10161/18362).

Data collection was led by Cassandra Nieman, Alexie Rudman, and Margaret Chory. Research was overseen by Grant Murray, Luke Fairbanks, and Lisa Campbell.

The research was conducted according to Duke University IRB protocol 2018-0427.

Data were collected June 2018 and November 2018, via informal interviews and observations, at two free fishing sites in Carteret County, North Carolina: the Newport River Pier and the Grayden Paul Drawbridge. Interviews were conducted at varied times throughout the day and on different days throughout the week. Everyone fishing at each study site when the researchers arrived was assigned a number based on their physical location, and a random number generator was used to select which respondents to interview. Using a "sample to saturation" strategy, interviewers collected information from multiple respondents until they were not gaining any new information. Between the two study sites, researchers approached 80 fishers and completed 74 semi-structured interviews.

Respondents were briefed on research objectives, and oral consent was obtained from interviewees. Interviews were guided by scripts, audio recorded, and transcribed. Demographic, quantitative (e.g. hours spent fishing), and some descriptive (e.g. species targeted) data were extracted from interviews and entered into an offline iPad survey form, to support a broad description of fishing activity.

Data are contained in two separate files, a PDF of interview transcripts and a CSV file of demographic, quantitative, and standardized response data.

Minor changes to the interview protocol were made as researchers gained familiarity with fishing practices and respondents. E.g. a question about whether or not people consumed their catch was expanded to ask how much of the catch was consumed. Additionally, changes were made when research transitioned to the fall and then again when the fall fishing season was disrupted by Hurricane Florence. E.g. questions were added about target species in different seasons; the impacts of Hurricane Florence on fishing; and the employment status of the respondent. 
All identifying information has been removed from the transcripts. Three transcripts are excluded in their entirety due to concerns about identifying information. For more information on interview coding and analysis, see description included with transcripts in the file Niemanetal_2021_FishingForFood_Interviews.pdf.

Data in the CSV file Niemanetal_2021_FishingForFood_Codedintervieweedata.csv should be re-used with caution. These data were extracted from informal interview text, rather than via a formal survey instrument. Responses were sometimes incomplete, unclear, or contradictory. Missing data (e.g. if respondent was not asked or did not answer a question) is labelled NR. If a question was not applicable (e.g. based on a prior response), the spreadsheet entry is NA. For questions that were not included in the interview at the time is was completed (see above description of changes to interview protocol), the spreadsheet entry is 'not asked.'

Interviewees were assigned a code, used in both transcripts and the spreadsheet. They are distinguished by season (with an "S" if conducted in summer, an " $F$ " if conducted in the fall) and location (with an "A" if conducted at the Newport River Pier, and a "B" if conducted at the Grayden Paul Drawbridge).

\section{Funding:}

This research was supported by NC Sea Grant project "The Value of Fishing, Farming, and Eating: new approaches to understanding coastal community well-being in a changing world", Project Number R/18-RCE-1, PI Grant Murray. Additional funding was provided by Duke University's Ramus Fund. Neither funding source was involved in the study design, collection and interpretation of the data, the writing of the report, or the decision to submit for publication. 